1) If deteriorating patients are recognised by nursing staff on the wards

2) If these patients are assessed promptly and by the appropriate professional

3) If escalation plans exist to guide on call doctors when making management plans for these patients.

Methods Data was retrospectively collected using a modified audit tool based on NICE 50 (Acutely ill patients in hospital). Patients at risk of deterioration were identified by an Early Warning Score $>5$. Thirty case notes were examined on several medical wards in Airedale General Hospital.

Results A doctor or member of the Outreach Team was contacted in $70 \%$ of cases when a patient had a high EWS $>5$ (standard 100\%). A third of patients were not reviewed after a high EWS (standard 100\%). Only 5 out of 30 patients had a clear escalation plan highlighting whether higher level care was appropriate. Evidence of preventable harm was not found but examples of poor practice were identified e.g. patients with palliative conditions in whom DNACPR had not been considered.

Discussion The results were presented orally to clinical staff and in the hospital newsletter to raise awareness of the need to make improvements. A distinctive Escalation Plan form and sticker was designed and is being piloted on the medical wards to encourage 'Day' teams to state a ceiling of care. Feedback will be obtained from staff when re-audit takes place.

Conclusion Assessment and management of deteriorating patients must be improved. With the above interventions we hope to show that this can be achieved in the DGH setting.

\title{
Ethics and Practice
}

\section{P 190 TO ESCALATE OR TO PALLIATE? AN AUDIT OF PATIENTS AT RISK OF DETERIORATION ON MEDICAL WARDS}

Bhajneek Grewal, Fiona Johnson. Airedale General Hospital, BD206TD, England

10.1136/bmjspcare-2014-000654.231

Background Junior doctors are often the first to assess deteriorating patients out of hours. From our experience, many deteriorating patients do not have escalation plans in place from the patient's regular medical team. Making decisions to escalate or palliate unwell patients is therefore made more challenging.

Objectives The primary objectives of this audit were to identify: 